\title{
Qualitätssicherung in der Psychiatrie
}

\author{
Das Internetportal Qualitätskliniken.de erweitert sein Angebot: Künftig soll es dort auch \\ Informationen über psychiatrische Abteilungen geben.
}

D ie Häufigkeit psychischer Erkrankungen nimmt zu. Das Klinikportal „qualitaetskliniken.de“ reagiert auf diese Entwicklung und dehnt sein Angebot jetzt auf die stationäre Psychiatrie aus. Binnen weniger Wochen wolle das Portal damit beginnen, Ergebnisse zur patienten- und einweiserbezogenen Qualität von stationären psychiatrischen Behandlungen in deutschen Kliniken zu veröffentlichen. Das haben Vertreter des Internetportals beim 3. Forum Qualitätskliniken mitgeteilt. Auslöser für die Erweiterung des Angebots seien die zunehmende Bedeutung der psychischen Erkrankungen und die geplanten gesetzlichen Änderungen. So habe die Zahl der Arbeitsunfähigkeitstage von Arbeitnehmern aufgrund psychischer Erkrankungen massiv zugenommen, sagte der Geschäftsführer der 4QD - Qualitätskliniken GmbH, Dr. Klaus Piwernetz. Um den daraus erwachsenden medizinischen und wirtschaftlichen $\mathrm{He}$ rausforderungen zu begegnen, regele die Bundesregierung derzeit die Vergütung von Behandlungen in psychiatrischen und psychosomatischen Einrichtungen neu.

\section{Mehr Transparenz nötig}

Mit dem so genannten Psychentgelt-Gesetz will die Regierung nicht nur ein pauschalierendes Entgeltsystem einführen. Der Entwurf soll gleichzeitig Grundlagen für eine systematische Qualitätssicherung in der psychiatrischen und psychosomatischen Versorgung schaffen, lauten die Pläne des Bundeskabinetts. Die Ministerrunde hat den Gesetzentwurf bereits genehmigt. Der Gemeinsame Bundesausschuss hat das Göttinger Aqua-Institut am 15. März damit beauftragt, ein sektorenübergreifendes Qualitätssicherungsverfahren für die Versorgung psychisch Erkrankter zu entwickeln. Dies wird aber voraussichtlich erst ab 2017 greifen. Bis dahin gilt die Qualitätssicherung in der Psychiatrie als unterentwickelt.

„Es muss dringend mehr Transparenz in die psychiatrischen Kliniken", forderte Dr. Iris Hauth vom Sankt Joseph-Krankenhaus in Berlin bei einem Pressegespräch des „Forums Qualitätskliniken“ in Berlin. Hier setzt die Initiative der Qualitätskliniken an. Das Portal werde ab Mitte 2012 Daten zur Patientensicherheit sowie zur Zufriedenheit von Patienten und einweisenden Ärzten und Therapeuten veröffentlichen, sagte Piwernetz. Diese Informationen seien für Angehörige, die Mitarbeiter von Krankenkassen und Privatversicherern, für die zuweisenden Ärzte und vor allem für die Patienten selbst zugänglich. „Mit der Vergleichbarkeit psychiatrischer Angebote wird die Rolle des Patienten gestärkt - und damit nicht zuletzt ein Beitrag zur Entstigmatisierung psychiatrischer Therapien geleistet", sagte Piwernetz.

„90\% der Psychiatriepatienten wählen ihre Klinik selbst aus“, erläuterte Dr. Roland Dankwart. „Dafür brauchen sie Instrumente wie die von qualitätskliniken. de bereit gestellten“, sagte der Konzerngeschäftsführer der Asklepios Kliniken $\mathrm{GmbH}$.

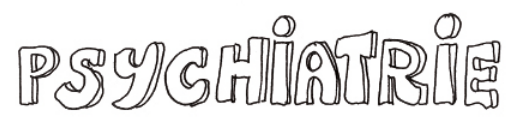

Einen herausragenden Stellenwert bei der Beurteilung psychiatrischer Kliniken und Fachabteilungen nähmen Informationen zur Patientensicherheit ein, berichten Versicherungsfachleute. „Stürze, Verletzungen der Aufsichtspflicht, falsch durchgeführte Therapien, fehlerhafte Medikationen und Überwachungsfehler sind die häufigsten Fehler, die in einem kausalen Zusammenhang zu Schadensmeldungen stehen", das hat eine Auswertung des Ecclesia Versicherungsdienstes ergeben.

\section{Fokus auf Prozess- und Ergebnisqualität}

Chefärztin Hauth plädierte dafür, der Prozess- und Ergebnisqualität großes Gewicht einzuräumen. Kriterien wie die Einhaltung und Integration von Leitlinien in die Patientenbehandlung seien dafür gute Ansätze. Auch ethische Fragen, wie das Personal von psychiatrischen Einrichtungen mit Zwangsmaßnahmen umgehe, sollten berücksichtigt werden.

„Qualitaetskliniken.de“ informiert unter anderem über Behandlungsqualität, Patientenzufriedenheit und -sicherheit. Das Klinikportal bezeichnet sich als unabhängig, trägerübergreifend und werbefrei. Träger ist die „4QD - Qualitaetskliniken.de $\mathrm{GmbH}$ “, hinter der die privaten Klinikunternehmen Asklepios, Rhön und Sana sowie der Zweckverband

freigemeinnütziger Krankenhäuser Münsterland und Ostwestfalen sowie der Zweckverband der Krankenhäuser Südwestfalen stehen.

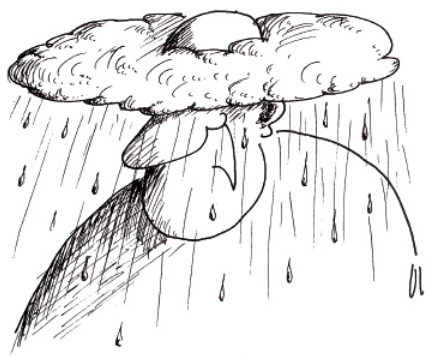

Pressegespräch „Qualität und Transparenz in der Psychiatrie", im Rahmen des 3. Forums Qualitätskliniken, 2. Mai 2012, Berlin af/sun 